\title{
Evaluation of Ceftriaxone utilization among selected hospitals in Dar-es- Salaam, Tanzania
}

\author{
${ }^{*}$ Mlugu Eulambius ${ }^{1}$, Nelson Osward ${ }^{1}$ \\ ${ }^{1}$ Department of Pharmaceutics, School of Pharmacy; Muhimbili University of Health and Allied Sciences, Tanzania
}

\section{ABSTRACT}

Objectives: Inappropriate use of ceftriaxone has been reported to increase treatment cost among patients. In addition, inappropriate use is a threat for resistance development. We assessed utilization of ceftriaxone among selected public and private hospitals in Dar es Salaam Tanzania.

Methods: Retrospective study was conducted from February to May 2017. Total of 600 patients' medication records were included. Chi square test was used to compare appropriateness use of ceftriaxone between the hospitals. Significance level was set at 0.05 and the confidence level at $95 \%$.

Results: Total of 330 (55\%) and 270 (45\%) patients were prescribed ceftriaxone for pre-operative prophylaxis, and treatment respectively. In overall, ceftriaxone was significantly utilized inappropriately in private hospital (72.3\%) than in public hospital (60\%) p=0.002 against the Tanzania standard treatment guideline. Ceftriaxone was more used inappropriately in private $(87 \%)$ than the public hospital $(69.2 \%)$ p $<0.001$ for prophylaxis. In contrary, for treatment indication ceftriaxone was more used inappropriately in public hospital (53.3\%) than in private hospital (38.9\%) $\mathrm{p}=0.026$.There were no records of sensitivity test in all the patients' files.
\end{abstract}

Conclusion: We report inappropriately utilization of Ceftriaxone against the Tanzanian standard treatment guideline. Private hospital was found to utilize ceftriaxone more inappropriately than the public hospital. We recommend further prospective studies involving all antimicrobials to be done.

Keywords: Ceftriaxone utilization, antimicrobial resistance, inappropriate use

Article Info: Received 03 Sep 2019; Review Completed 02 Oct 2019; $\quad$ Accepted 10 Oct 2019; $\quad$ Available online 15 Nov 2019

\section{Cite this article as:}

Eulambius M, Osward N, Evaluation of Ceftriaxone utilization among selected hospitals in Dar-es-Salaam, Tanzania, Journal of Drug Delivery and Therapeutics. 2019; 9(6):7-11 http://dx.doi.org/10.22270/jddt.v9i6.3662

\section{*Address for Correspondence:}

Tanzania

Mlugu Eulambius, Department of Pharmaceutics, School of Pharmacy; Muhimbili University of Health and Allied Sciences,

\section{INTRODUCTION}

Since their discoveries antimicrobials have transformed global healthcare and saved millions of lives. Currently the world is threatened by the growing burden of antimicrobial resistance. Common curable infections are fast becoming deadly because antibiotics no longer respond to the infections that use to respond before 1 . In the United States it is estimated that each year at least 2 million people acquire serious infections with bacteria that are resistant to one or more of the antibiotics designed to treat those infections and that at least 23,000 people die each year as a result of antibiotic-resistant infections ${ }^{2}$. World Health Organization(WHO) cautions that antimicrobial resistance will increase healthcare costs for individuals, health systems, and countries if actions will not be taken ${ }^{3}$. Use, overuse and misuse of antimicrobials are the reasons for the developing resistance ${ }^{4}$. The current antimicrobial action plan stipulates optimization of antimicrobial agents as one of the strategies for fighting against antimicrobial resistance. Drug use evaluation studies have been good approaches for promoting the quality and optimization of drug care in the treatment centers.

Ceftriaxone is a third generation bactericidal Cephalosporin antibiotic with wide spectrum activity and good safety profile. It is widely used to treat various bacterial infections including bronchitis, pneumonia, bone infections, abdominal infection, skin infections, meningitis, endocarditis, urinary tract infections, septicemia and surgical prophylaxis ${ }^{5}$. Due to its high antibacterial activity, extensive spectrum of activity, low potential for toxicity it is one of the most widely used antibiotics 6,7 .

Evidence from both developed and developing countries indicates inappropriate utilization of ceftriaxone. In developed countries inappropriate use of ceftriaxone is not as worse as in developing countries. For instance in Korea a study found only $34.5 \%$ of inappropriate use 8 .The study in 
tertiary hospital in Port of Spain found ceftriaxone as the most prescribed cephalosporin antibiotic ${ }^{9}$. In contrary, two studies in Ethiopia found inappropriate use of ceftriaxone as high as $70 \%$ and $80 \%$ respectively 10,11 . Inappropriate use of ceftriaxone does not only increase treatment cost to patients but also may put selective pressure on bacteria to acquire resistance genes hence result in emergence of antibiotic resistance.

Tanzania is one of the resources limited countries with high demand for antibiotics influenced by the high burden of infectious disease. Inappropriate use of ceftriaxone is likely to happen. Unfortunately there is limited data, thus it is not known to what extent is the problem. Data on utilization of ceftriaxone are very important especially currently where implementation of global antimicrobial resistance action plan is on the table. Therefore the present study gives an insight regarding utilization of ceftriaxone among selected public and private hospitals in Dar es salaam, Tanzania. These findings may pave the way for further studies to evaluate antimicrobial use in health facilities as the basis for their rational use.

\section{METHODS}

\section{Study design and area}

A retrospective cross sectional study was conducted from February to May, 2017. The study reviewed medical records of patients previously admitted at Mwananyamala (public hospital) and Tumaini (private hospital) in Dar es Salaam Tanzania. The hospitals were purposively selected.

\section{Inclusion and exclusion criteria}

This study included medical records of all patients previously admitted in medical and surgical wards and stayed for more than 24 hours. Patients who had received at least one course of any dose of ceftriaxone treatment from January to December 2016 were included. Medication records that were incomplete in terms of main variables were excluded.

\section{Sample size and selection}

A WHO guideline for the study of medication use was used to obtain the representative sample size. A total of 600 (300 from each hospital) patients medication records were purposively selected.

\section{Data collection procedure}

A pre-tested checklist contained information on gender, age, dose, frequency of administration, number of co-morbid conditions, number and type of co-prescribed medications and duration of ceftriaxone therapy was used for data collection. The files of patients treated with ceftriaxone were traced back to the records of the hospitals using the card numbers obtained from the inpatient registration documents. Data collection format was developed based on the WHO criteria for Drug Use Evaluation. Tanzanian Standard Treatment Guideline and National essential medicine list of $2013^{12}$ was used to validate the tool for ceftriaxone use evaluation. Inappropriate use of ceftriaxone in the hospitals was determined by comparing the observed ceftriaxone prescribing in the patient's file to the recommendations in the Tanzania national standard treatment guideline.

\section{Ethics consideration}

Ethics approval was granted by the Institutional review board of the Muhimbili University of Health and Allied Sciences (MUHAS). Permission to conduct the study in the selected health facilities was sought and obtained from the Medical Officers in charge prior data collection. In order to ensure confidentiality of the study participants' data were entered in the computer and analyzed using code numbers instead of names.

\section{Data management and analysis}

The coded data were checked and cleaned before entered in to Statistical package for social sciences (SPSS) version 20 for analysis. Descriptive statistics and crosstabs were employed to assess adherence to ceftriaxone utilization. Chi square test was used to compare ceftriaxone use adherence between public and private hospitals. Significance level was set at 0.05 and the confidence level at $95 \%$.

\section{RESULTS}

A total of 600 (300 from public hospital and 300 from private hospital) patients' records were included in this study. In both hospitals majority of participants (58.7\%) had ages below 35 years. Few participants in public hospital had age beyond sixty years but in private hospital about quarter of participants had the age beyond sixty years. In both hospitals majority $(57 \%)$ of all participants were females (Table 1 ).

Table 1: Socio-demographic characteristics of participants $(n=600)$

\begin{tabular}{lll}
\hline Characteristics & Public hospital n (\%) & Private hospital n(\%) \\
\hline Age (in years) & $28(2.5)$ & $32(3.6)$ \\
Mean age (SD) & $195(65)$ & $157(52.3)$ \\
$14-35$ & $82(27.3)$ & $69(23)$ \\
$36-60$ & $23(7.7)$ & $74 \quad(24.7)$ \\
$\geq 60$ & & $135(45)$ \\
Sex & $122(40.7)$ & $165(55)$ \\
Male & $178(59.3)$ & $210(63.6)$ \\
Female & $120(36.4)$ & $90(33.3)$ \\
Prophylaxis indication of ceftriaxone & $180(66.7 \%)$ & \\
Therapeutic indication of ceftriaxone & & \\
\hline
\end{tabular}




\section{Common indications of ceftriaxone in the hospitals}

A total of 330 patients (55\%) (120 from public hospital and 210 from private hospital) were prescribed ceftriaxone for pre-operative prophylaxis. Two hundred and seventy (45\%) patients (180 from public hospital and 90 from private hospital) were prescribed ceftriaxone for the treatment of bacterial infection. Ceftriaxone was prescribed for treatment of Pneumonia, Septicemia, urinary tract infection, bacterial meningitis, chronic kidney diseases (CKD), and pelvic inflammatory disease (PID). About $16.4 \%$ of the prescriptions were for the conditions not indicated in the standard treatment guideline. In terms of indication Pneumonia was the most common disease treated by ceftriaxone (Figure 1).

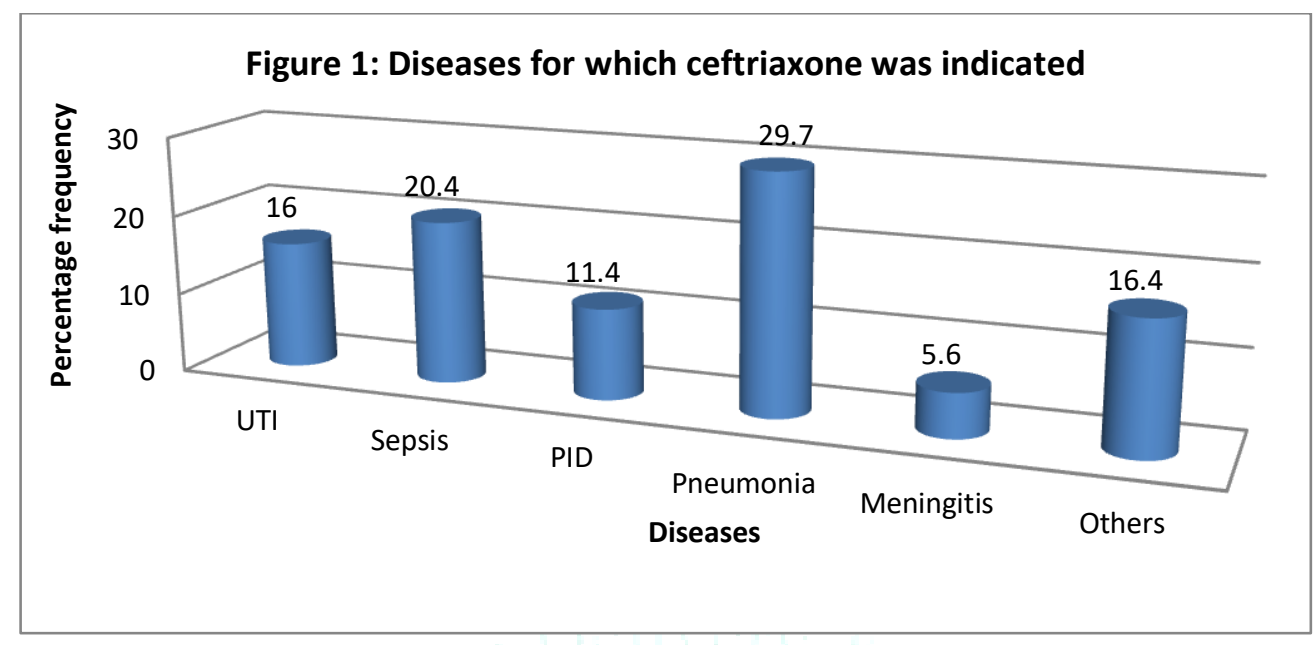

\section{Overall standard treatment compliance of ceftriaxone therapy}

In overall, ceftriaxone was significantly inappropriately prescribed in private hospital (72.3\%) than in public hospital $(60 \%) \mathrm{p}=0.002$. In pre-surgical prophylaxis, ceftriaxone was significantly inappropriately used in private hospital (87\%) than the public hospital (69.2\%) p=0.0001 (Table 2). The inappropriate prescribing was due to longer duration of administration (3-5days) than which is stated in the Standard Treatment guideline. Among patients prescribed ceftriaxone for treatment of bacterial infections, inappropriate use was significantly more in the public hospital than in private hospital. Inappropriate use was for the duration prescribed, frequency and wrong indication. Ceftriaxone compliance with Tanzania standard treatment guideline was more for pre-surgery prophylaxis than in treatment of bacterial infections in all the hospitals (Figure 2).

Table 2: Ceftriaxone utilization; Standard treatment guideline compliance between the two hospitals

\begin{tabular}{|c|c|c|c|}
\hline Prescribing pattern & $\begin{array}{l}\text { Public Hospital n } \\
(\%)(n=300)\end{array}$ & $\begin{array}{l}\text { Private hospital } n \\
(\%)(n=300)\end{array}$ & P-value \\
\hline \multicolumn{4}{|l|}{ Overall STG compliance } \\
\hline Appropriate utilization & $121(40)$ & $83(27.7)$ & \\
\hline Inappropriate utilization & $179(60)$ & $217(72.3)$ & 0.002 \\
\hline \multicolumn{4}{|c|}{ STG prophylactic use compliance } \\
\hline Appropriate utilization & $37(30.8)$ & $28(13)$ & \\
\hline Inappropriate utilization & $83(69.2 \%)$ & $182(87 \%)$ & $<0.0001$ \\
\hline \multicolumn{4}{|c|}{ STG therapeutic use compliance } \\
\hline Appropriate utilization & $84(46.7)$ & $55(61.1)$ & \\
\hline Inappropriate utilization & $96(53.3 \%)$ & $35(38.9 \%)$ & 0.026 \\
\hline
\end{tabular}




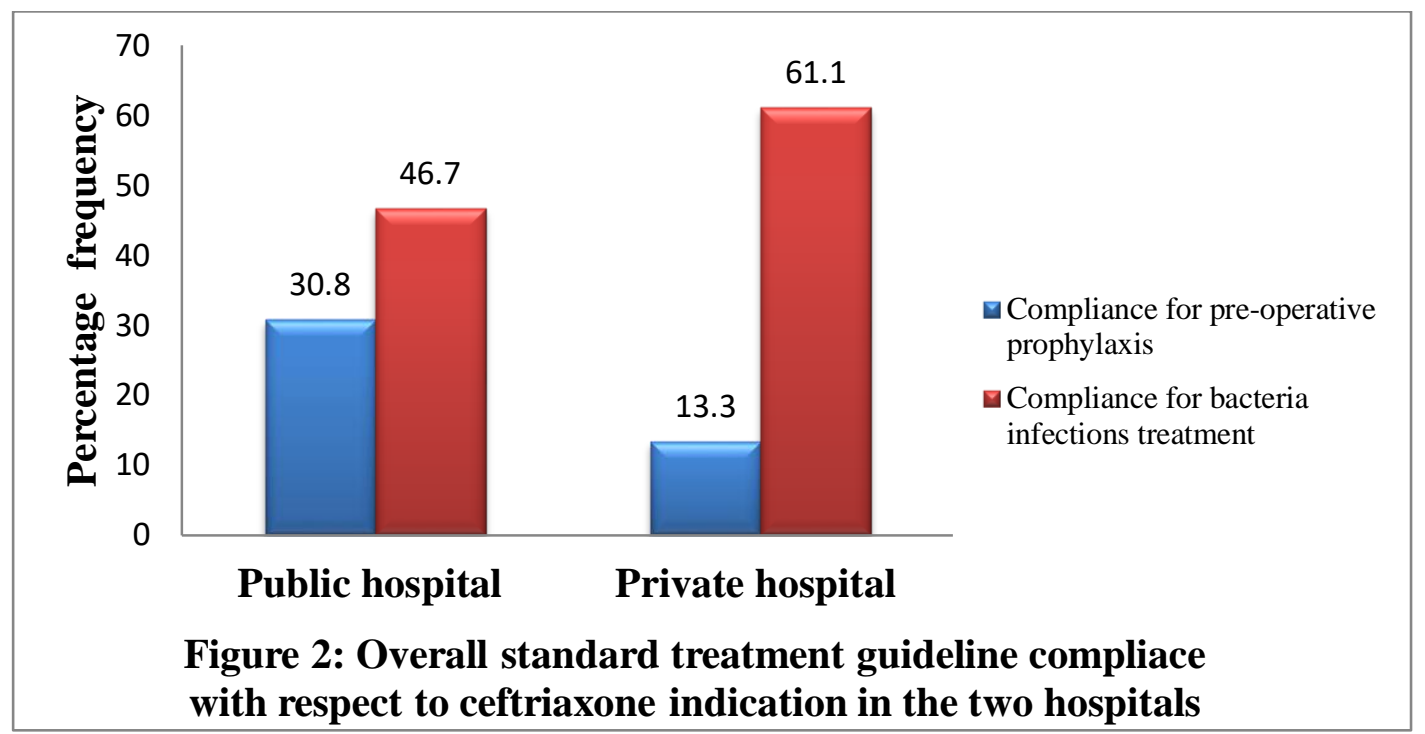

\section{DISCUSSION}

We report ceftriaxone utilization in selected public and private hospitals in Dar es Salaam, Tanzania. The major findings of this study were, (i) overall poor adherence to Tanzania standard treatment guideline $(>50 \%)$ with regard to ceftriaxone utilization in both hospitals,(ii) ceftriaxone was commonly used for pre-operative prophylaxis, and (iii) pneumonia being the most common indication where ceftriaxone was prescribed.

High inappropriate utilization of ceftriaxone found in this study indicates a problem in rational use of ceftriaxone in the hospitals. Inappropriate use of ceftriaxone indicates negligence to standard treatment guideline adherence. In addition, the high inappropriate utilization pose threats for resistance development. There may be a challenge of standard treatment guideline availability and use in both hospitals which may have been contributed to the findings. It is important that emphasis on the importance of adhering to STG become the priority among healthcare workers especially for antibiotics bearing in mind the globe threat to antimicrobial resistance. In addition the ministry responsible for health should make sure that STGs are made available and utilized to all health facilities The findings are similar to the findings from Ethiopia 13, 14 indicating a challenge in sub-Saharan Africa.

Inappropriate use of ceftriaxone was significantly found to be more in private hospital than in public hospital. This difference might have been due to less availability of STGs in private hospitals than in public hospital although this was not part of this study. Influence by profit making in private setting than in public setting may also contribute to the observed difference. Regardless of the hospital setting rational use of antibiotics need to be the agenda to all health facilities. These results are similar to the comparative retrospective study between public and private hospital done by Tariku et.al in Ethiopia 15.

In this study ceftriaxone was found to be commonly used for preoperative prophylactic. More inappropriate use of ceftriaxone in this indication was detected in both hospitals although adherence to STG was significantly more in public hospital than in private hospital. According to Tanzania Standard Treatment guideline normally prophylactic antibiotic should be used 30 minutes or 1 hour before the surgical procedure and should be used not more than 24 hours unless susceptible bacterial infections diagnosed. We found ceftriaxone prescribed between 3 to 7 days presurgery without any susceptible bacterial infection recorded contrary to Tanzania standard treatment guideline. This unnecessary use of ceftriaxone adds on treatment costs to patients and may put pressure on this potential antibiotic for resistance development.

Ceftriaxone was also found to be prescribed for treatment of various bacterial infections. In this case ceftriaxone was found to be used inappropriately significantly more in the public based hospital than in private hospital. The inappropriate uses were due to wrong duration of treatment, wrong frequency, wrong dose and wrong indication according to Tanzania standard treatment guideline. Although ceftriaxone was prescribed appropriately for treatment of pneumonia, sepsis, Urinary tract infection, Pelvic inflammatory disease, and meningitis, a significant number of inappropriate uses are of concern. For instance, contrary to Tanzania standard treatment guideline, ceftriaxone was prescribed to diseases such as chronic obstructive pulmonary disorders (COPD), dyspepsia, typhoid fever, chronic kidney diseases (CKD) and cardiovascular diseases without any record of co-morbidity requiring such a prescription. This contributed to more than sixteen percent of all the ceftriaxone prescriptions used for therapeutic. Moreover there no any record of bacteria susceptibility test requested in all patients. Pneumonia was the leading disease where ceftriaxone was mostly prescribed. These findings are similar to those of Getasewet $a l$ in Dessie referral hospital Ethiopia ${ }^{16}$.

The limitation for this study could be the fact that it was a retrospective that only assessed records of patients who were prescribed ceftriaxone thus we couldn't monitor treatment outcomes. Some of the information also might have been not reported in the patient's records thus the actual inappropriate use of ceftriaxone could have been underestimated or overestimated.

\section{CONCLUSION}

Ceftriaxone was mostly found to be utilized inappropriately against the Tanzanian treatment guideline. Private hospital was found to utilize ceftriaxone more inappropriately than the public hospital. We recommend further prospective study involving all antimicrobials to be done. 


\section{ACKNOWLEDGEMENT}

We convey our sincere thanks to management of Mwananyamala and Tumaini hospitals for accepting our request to undertake this study. Our thanks also go to healthcare workers in the two hospitals especially workers of records unit for their tireless support during data collection.

\section{Conflict of interest}

The authors declare that they have no any competing interest

\section{Funding}

This study did not receive any specific funding.

\section{REFERENCES}

1. Ventola, C.L., The antibiotic resistance crisis: part 1: causes and threats. 2015. 40(4): p. 277-83.

2. CDC. Antibiotic Use in the United States, 2017: Progress and Opportunities. Atlanta, GA: US Department of Health and Human Services, CDC; 2017.

3. World Health Organization. Antimicrobial resistance, Global report on surveillance 2014. Geneva 2014, 2014.

4. Joseph, N.M., et al., Relationship between Antimicrobial Consumption and the Incidence of Antimicrobial Resistance in Escherichia coli and Klebsiella pneumoniae Isolates. Journal of clinical and diagnostic research : JCDR, 2015. 9(2): p. DC08DC12.

5. Petri WA. Penicillins, Cephalosporins, and Other $\beta$-Lactam Antibiotics. In: Brunton LL, Chabner BA, BC K. Goodman \& Gilman's the pharmacological basis of therapeutics. $12^{\text {th }}$ ed. New York: McGraw-Hill; 2011

6. Fahimzad, A., et al., Surveillance of Antibiotic Consumption Point Prevalence Survey 2014: Antimicrobial Prescribing in Pediatrics Wards of 16 Iranian Hospitals. Arch Iran Med, 2016. 19(3): p. 204-9.
7. Iskandar, K., et al., Antibiotic consumption in non-teaching Lebanese hospitals: A cross-sectional study. Journal of Infection and Public Health, 2016. 9(5): p. 618-625.

8. Lee, H., et al., Evaluation of ceftriaxone utilization at multicenter study. The Korean journal of internal medicine, 2009. 24(4): p. 374-380.

9. Pinto Pereira, L.M., et al., Third generation cephalosporin use in a tertiary hospital in Port of Spain, Trinidad: need for an antibiotic policy. BMC Infectious Diseases, 2004. 4(1): p. 59.

10. Ayele, A.A., et al., Prospective evaluation of Ceftriaxone use in medical and emergency wards of Gondar university referral hospital, Ethiopia. Pharmacology research \& perspectives, 2018. 6(1): p. e00383.

11. Negese S. G, M.A., Amsale M, Asmamaw D, Omer M, Tenaw A. Evaluation of Ceftriaxone Use for Hospitalized Patients in Ethiopia: The Case of a Referral Hospital. IJPSR, 2017. 3:2.

12. United republic of Tanzania. Standard Treatment Guidelines and Essential Medicines List. Ministry of Health and Social Welfare. Fourth Edition, 2013.

13. Sileshi, A., et al., Evaluation of ceftriaxone utilization in medical and emergency wards of Tikur Anbessa specialized hospital: a prospective cross-sectional study. BMC pharmacology \& toxicology, 2016. 17: p. 7-7.

14. 14. Hafte K, T.K., Azeb W, et al., Assessment-of-ceftriaxoneuse-in-eastern-ethiopian-referral-hospital-a-retrospectivestudy. J Infectious Disease Med Microbiol. 2018;2(3):26-9.

15. Shimels, T., A.I. Bilal, and A. Mulugeta, Evaluation of Ceftriaxone utilization in internal medicine wards of general hospitals in Addis Ababa, Ethiopia: a comparative retrospective study. Journal of pharmaceutical policy and practice, 2015. 8: p. 26-26.

16. Ayinalem, G., et al., Drug use evaluation of ceftriaxone in medical ward of Dessie Referral Hospital, North East Ethiopia. International Journal of Basic \& Clinical Pharmacology, 2013. 2(6): p. 711. 